\title{
Emotion Mirror: A novel intervention for autism based on real-time expression recognition
}

\author{
David Deriso $^{1}$, Joshua Susskind ${ }^{1}$, Lauren Krieger $^{2}$, Marian Bartlett ${ }^{1}$ \\ ${ }^{1}$ Institute for Neural Computation, University of California, San Diego \\ ${ }^{2}$ Dept of Film, Television, and Digital Media, University of California, Los Angeles \\ \{deriso,jmsusskind, laurenkriegerart\}@gmail.com, mbartlett@ucsd.edu
}

\begin{abstract}
Facial expression perception and production are crucial for social functioning. Children with autism spectrum disorders (ASD) are impaired in their ability to produce and perceive dynamic facial expressions, which may contribute to social deficits. Here we present a novel intervention system for improving facial expression perception and production in children with ASD based on computer vision. We present a live demo of the Emotion Mirror, a game where the children make facial expressions of basic emotions (anger, disgust, fear, happiness, sadness, and surprise) that are "mirrored" by a cartoon character on the screen who responds dynamically in real-time. In the reverse mirror condition, the character makes an expression and children are rewarded when they successfully copy the expression of the character. This application demonstrates a novel intersection of computer vision and medicine enabled by real-time facial expression processing.
\end{abstract}

\section{Introduction}

Recent advances in automated facial expression recognition technology open new possibilities for clinical research, including assessment and interventions. Children with autism spectrum disorders (ASD) are impaired in their ability to produce [1] and perceive dynamic facial expressions [2]. This demo stems from a multidisciplinary research effort to develop and evaluate a computer assisted intervention system to enhance the facial expression skills of children with ASD that merges the expertise of researchers in computer vision, face perception, autism, and social neuroscience. Advanced computer vision technology can now be leveraged in the investigation of the facial expression perception and production deficits in children with ASD. This technology can be used to quantify deficits in a way that was impractical with previously existing methods, and to create real-time interventions aimed at reducing production and perception deficits.

Here we present a live demo of an intervention game developed for this project called the Emotion Mirror. This is an intervention focused on expression production, which provides real-time feedback on the child's own expressions. The goal is to improve the child's facial expression displays, and also to link motor movement with perception in order to recruit the motor system in the recognition and understanding of the emotion. A body of 
research in cognitive neuroscience has linked expression perception with motor production systems in the brain, and it has been proposed that a dysfunction in the mirror neuron system may underlie social deficits in autism. e.g. [3].

\section{The Computer Expression Recognition Toolbox}

The facial expression recognition engine behind the Emotion Mirror is the Computer Expression Recognition Toolbox (CERT) [4]. CERT is a fully automated system for measuring facial expressions in real time [4]. CERT automatically codes the intensity of 6 expressions of basic emotion, 3 dimensions of head pose (yaw, pitch, and roll, as well as the 20 facial actions from the Facial Action Coding System (FACS) [5] most related to emotion. FACS is a system for objectively coding facial expressions in terms of elemental movements, called action units (AUs), which roughly correspond with individual facial muscle movements. The technical approach to CERT is an appearance-based discriminative approach. Such approaches have proven highly robust and fast for face detection and tracking (e.g. [6]), and have enabled real-time facial expression applications. See [4] for more information on system design and benchmark performance measures. CERT operates in real time at approximately 12-15 frames per second, and is available for academic use.

\section{The Emotion Mirror}

The emotion mirror is an intervention game in which an avatar responds to facial expression of the subject in real-time. In one condition, the character mirrors the participant's expression ('it mimics you'). The participant is prompted to make one of 6 expressions, and when the expression is successfully produced, the character mirrors the participant's expression. In the reverse condition ('you mimic it') the subject copies the character's expression. As the participant approximates the expression of the character, an ice-cream cone grows by increasing in units of scoops and toppings. A softmax competition between CERT's six expressions of basic emotion and neutral mediates the matching signal between the prompted emotion and that of the participant. This matching signal drives the character's expression intensity in 'it mimics you' and the height of the ice cream in 'you mimic it' in proportion to the participant's expression intensity.

The game includes a selection of engaging animated characters that range in visual complexity. It is well known that children with ASD tend to be more comfortable with visually simple displays, and are more comfortable with robots and animals than with human faces. The avatars in the intervention game therefore range from animals, to outer-space creatures, to a more realistic human avatar for which children can choose gender, skin color, and hair color to match their own. See Figure 2. The task knits expressive production and perception as it is the participant's own face that drives the expressions shown on the avatar, and provides feedback and practice in facial expression mirroring behaviors. 

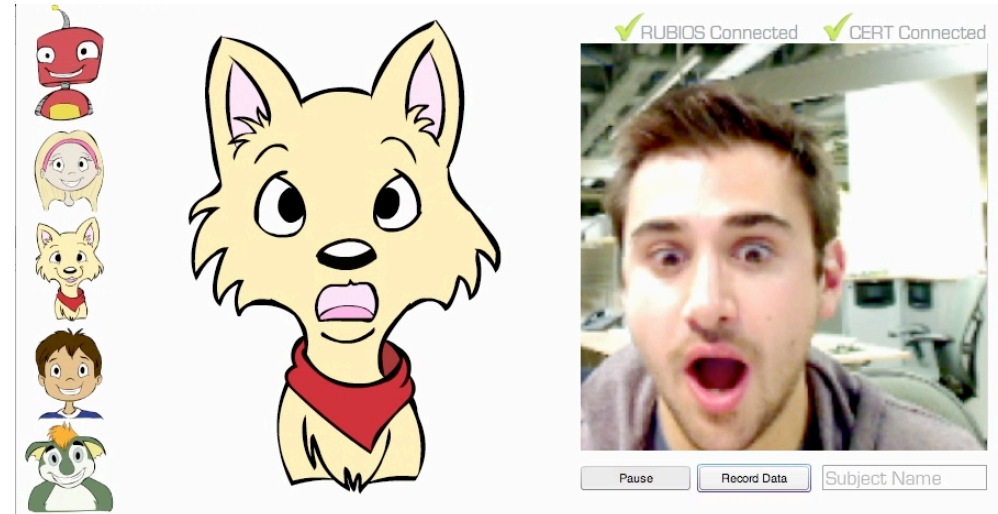

Fig 2. Emotion Mirror: An avatar responds to facial expressions of the subject in real-time.

\section{$4 \quad$ Intervention Design}

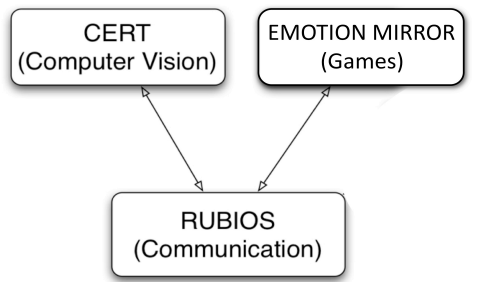

Fig 1. Structure of the software components of the intervention platform.

The intervention design is a mobile, low cost, platform to improve facial expression production in children with ASD. The hardware for the platform consists of a laptop computer equipped with an integrated Web camera. The software components for the intervention platform consist of (1) CERT (Computer Expression Recognition Toolbox), a program for computer vision based recognition of facial expressions, (2) EMOTION MIRROR: An intervention game written in Adobe Flash, (3) RUBIOS: a library of message passing functions to facilitate inter-process communication between the different software components (Figure 1).

\section{Discussion}

Recent advances in computer vision open new avenues for computer assisted intervention programs that target critical skills for social interaction, including the timing, morphology and dynamics of facial expressions. Such systems enable investigations into the learning of facial expression production that were previously not possible. The Emotion Mirror presents pioneering work to develop an engaging intervention system for facial expression 
processing based on automatic facial expression recognition technology. Importantly, this intervention links motor production with perception in a real-time feedback system.

Expression understanding may span multiple brain structures [7], which must communicate and pass activation through time. Disorders of temporal processing at any level of this system, whether it is in perception, production, or passing of activation between brain structures, could disrupt this system and hence disrupt emotion understanding. Temporal processing deficits have been demonstrated in autism, and may underlie a range of impairments [8]. Together, this suggests that social processing interventions should tap multiple processes, not individually, but together and at the right temporal intervals. Recent technology from computer vision and animation opens up new possibilities for intervention systems that link perception, production, and reward on timescales related to social responding. Such technology contributes not only to potential treatments, but also to the study of learning and plasticity in perception and production systems, and to understanding the cognitive neuroscience of emotion [9].

Acknowledgements. Support for this work was provided by NIH grant NIMH- RC1 MH088633 and NSF grant SBE-0542013. Any opinions, findings, conclusions or recommendations expressed in this material do not necessarily reflect the views of the National Science Foundation.

\section{References}

1. McIntosh, D., et al., When the social mirror breaks ... Developmental Science. Developmental Science, 2006. 9: p. 295-302.

2. Adolphs, R., L. Sears, and J. Piven, Abnormal processing of social information from faces in autism. J Cog Neuroscience, 2001. 13: p. 232-240.

3. Williams, J., et al., Imitation, mirror neurons and autism. Neuroscience \& Biobehavioral Reviews, 2001. 25( 4): p. 287-295.

4. Littlewort, G., et al., The computer expression recognition toolbox (CERT), in IEEE International Conference on Automatic Face and Gesture Recognition. 2011, IEEE Xplore. p. 298 - 305.

5. Ekman, P. and W. Friesen, Facial Action Coding System: A Technique for the Measurement of Facial Movement. 1978, Palo Alto, CA: Consulting Psych. Press.

6. Viola, P. and M. Jones, Robust real-time face detection. J Computer Vision, 2004. 57(2): p. 137-154.

7. Niedenthal, P.M., Mermillod, M., Maringer, M. \& Hess, U., The Simulation of Smiles (SIMS) Model:Embodied Simulation and the Meaning of Facial Expression. Behavioral and Brain Sciences, 2010. 33(6): p. 417-433.

8. Gepner, B. and F. Féron, Autism: a world changing too fast for a mis-wired brain? Neurosci Biobehav Rev, 2009. 33(8): p. 1227-42.

9. Bartlett, M., Emotion simulation and expression understanding: A case for time. Invited commentary on Niedenthal et al. Behavioral and Brain Sciences, 2010. 33(6): p. 435-436. 